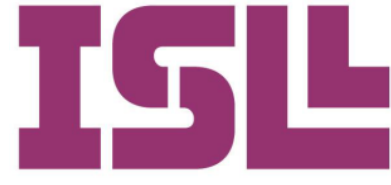

Recibido:

Noviembre 2013

Aprobado:

Febrero 2014

Pág. 32 a la 43

\section{Caracterización de las competencias transversales. La competencia lectora: identificación del nivel de logro}

\author{
Claudia Edith Ostria Baltazar \\ ITESM Campus Ciudad de México \\ María Guadalupe López Padilla \\ ITESM/Universidad Regiomontana
}

Jaime Ricardo Valenzuela González

ITESM

Palabras clave
competencias,
lectura,
características,
nivel.

Keywords

skills, reading, characteristics, level.

\section{Resumen}

El estudio indaga qué características distinguen a lectores competentes, mediante el paradigma postpositivista y la aplicación de los métodos mixtos para determinar el nivel de logro en estudiantes. Se realizó con escolares de primero y segundo grado de secundaria de la Ciudad de México. Primero se realizó un estudio cualitativo: seis entrevistas semiestructuradas a alumnos considerados por docentes, compañeros y ellos mismos, como competentes lectores. Ello permitió identificar cualidades de la competencia. En la segunda etapa, cuantitativa, con 70 alumnos, mediante instrumento auto-reporte, 40 ítems en escala Likert, se midieron cuatro dimensiones de la competencia lectora. Se analizaron resultados de entrevistas e instrumentos. En la cuantitativa: análisis descriptivo, psicométrico y de relaciones que permitieron observar el desarrollo de la competencia lectora para responder cuestionamientos motivo de investigación. Se obtuvieron resultados: identificación y caracterización de los buenos lectores. Las conclusiones sugieren mejorar y ampliar la investigación, así como un programa complementario.

\begin{abstract}
The main purpose of this investigation is to know what makes a competent reader, through post positivism paradigms and through some methods that were apply to determinate the level of improvement that was achieve by students. The target were kids of first and second grades of middle school in Mexico City. The first phase was a qualitative study: Six semi structured interviews to considered competent readers students. The second part was quantitative and it implied the autoreport instrument of 40 items in Likert scale to 70 students, to measure four dimensions of reading literacy. The final phase was to analyze the results of both strategies. Quantitative: Descriptive, psychometric and relation analysis to know the reader literacy development in order to answer questions. As well, results were obtained: identification and characterization of the good readers. Finally there are some conclusions to improve and amplify the investigation, as well as a complementary program.
\end{abstract}




\section{Introducción}

El presente estudio se propuso identificar y analizar las características particulares de la competencia lectora, circunscrita a un conjunto de competencias transversales, las cuales son necesarias para dar cumplimiento a cualquier currículo determinado. El cuestionamiento principal propuesto, razón de la investigación, es: ¿Qué cualidades o atributos distinguen a los alumnos de primer grado de secundaria que son lectores competentes de aquellos alumnos que no lo son?

Para ello, encuentra justificación en los resultados que arrojan un par de evaluaciones que ponen a México en un bajo nivel de comprensión lectora:

1. Resultados de la aplicación de la Prueba Program for International Student Assessment (PISA). En la aplicación del año 2009, el énfasis estuvo puesto en la competencia lectora. Los estudiantes mexicanos obtuvieron un promedio de 425 puntos, por debajo de los países participantes, cuya media se reporta en 493 puntos (Fundación Este País, 2011).

2. La Encuesta Nacional de Lectura: estudio realizado por la "Fundación Mexicana para el fomento de la lectura, A. C." en el año 2012.El 46\% de los mexicanos mayores de 12 años leen. Los libros leídos al año por persona son 2.94 en promedio. El 33\% de las personas que lee no tiene preferencias de lectura, el 26\% prefiere libros, el $18 \%$ prefiere leer el periódico y el $17 \%$ revistas. En la mayoría de los hogares mexicanos (87\%) se tienen entre 1 y 30 libros (FunLectura, 2012).

Ahora bien, la competencia lectora se ha definido como "la capacidad que tiene un individuo de comprender, utilizar y analizar textos escritos con objeto de alcanzar sus propias metas, desarrollar sus conocimientos y posibilidades y participar en la sociedad" (OCDE, 2006, p. 13).En esta definición, el énfasis está colocado en los conocimientos y las habilidades funcionales que hacen posible una participación activa en la sociedad, no limitada a la realización de tareas asignadas de manera externa sino que más bien implica que una persona está dotada de los recursos necesarios para participar en un proceso de toma de decisiones.

En palabras de León, Escudero y Olmos (2012) la competencia lectora es una perspectiva de la lectura en la que se da una serie de procesos de comprensión profunda (entendida a su vez como el esfuerzo por encontrar el significado), en donde se utiliza y se reflexiona sobre el contenido con la finalidad de alcanzar los objetivo y las metas que el lector se ha propuesto, de poseer más conocimientos y de ser más participativo socialmente.

En este sentido, leer implica comprender cualquier material escrito de cualquier naturaleza, extraer de él su significado y hacerlo consciente. El esfuerzo consiste en activar conjuntamente varios subprocesos, recurrir a conocimientos previos para contextualizar el contenido de la lectura, inferir información que aunque no esté directamente contenida en el texto, pueda ser referenciada a partir de los conocimientos que posee el lector. La 
competencia lectora alude "a todos los procesos mentales y las habilidades que se requieren para ser eficiente en la vida diaria” (León, Escudero y Olmos, 2012 p. 17).

La prueba estandarizada denominada PIRLS (Progress in International Reading Literacy Study) adopta una noción amplia de competencia lectora, a partir de tres aspectos: los procesos de comprensión que intervienen en la lectura, los propósitos de la lectura (disfrutar de la experiencia literaria y obtener información) y los hábitos y actitudes ante la lectura. Es decir, PIRLS define la competencia lectora como la construcción de significados que a partir de un texto escrito hace un alumno, mostrando la capacidad de realizar una serie de actividades o tareas cognitivas que se denominan procesos de comprensión. (Instituto Nacional de Evaluación Educativa, 2012).

\section{Método}

El estudio se enmarca dentro del paradigma postpositivista, debido a que éste nos permite mantener una idea objetiva a la que es preciso aspirar, revisando continuamente, modificando e inclusive retirando las afirmaciones que se hacen al enfrentarse a nuevas investigaciones (Valenzuela, 2011).

Por su naturaleza condicionada al marco teórico que lo rige, propone como metodología de trabajo de investigación, los métodos mixtos, con la intención de que los fenómenos propios de su estudio sean comprendidos con mayor riqueza, toda vez que recurren a la combinación de métodos cuantitativos y cualitativos (Valenzuela, 2011).

El estatus mediante el cual se trabajó la investigación es equitativo, se constituyó de manera uniforme la parte cualitativa y la cuantitativa en el estudio. Para Pereira (2011) la igualdad en el estatus se establece cuando no hay un estatus dominante, que ninguno de ellos se prioriza sobre el otro, solo varía el orden en cuanto a la secuencialidad.

Etapa 1: aproximación cualitativa.

Se empleó el método de investigación de casos múltiples. Con respecto a la obtención de datos, fueron obtenidos mediante una fuente cualitativa: entrevista directa.

a) Participantes: muestra no probabilística de 6 mediante el criterio bajo el cual fueron considerados por sus propios compañeros como personas muy competentes en materia de competencia lectora.

b) Instrumentos: el instrumento que se utilizó fue una entrevista semi-estructurada de veinte preguntas base, con la intención de recolectar los datos desde el contexto en el cual el fenómeno social ocurre de manera natural; esto permitió generar una comprensión basada en las perspectivas que se habían planteado (Martínez, 2006).

c) Procedimientos de colección de datos: por lo que respecta a los procedimientos, se planteó un protocolo de cuatro etapas (Martínez, 2006): 
1. Semblanza del estudio de caso. Selección de los casos de estudio, los participantes y se les invitó a participar del estudio.

2. Preguntas del estudio de caso. Elaboración del instrumento, estructurado a partir de la concepción de la competencia y del nivel de dominio que poseen las personas estudiadas.

3. Procedimientos a ser realizados. Se llevaron a cabo las entrevistas, las cuales fueron grabadas en audio y posteriormente transcritas para su análisis.

d) Procedimientos de análisis de datos: se realizó una guía del reporte del estudio de caso. Se analizaron los datos de la etapa 1, con la intención de establecer una base para constituir los elementos de investigación de la etapa cuantitativa.

Etapa 2: aproximación cuantitativa

En esta etapa se contrasta la teoría ya existente a partir de una serie de hipótesis surgidas de ella, para lo cual es imprescindible conseguir una muestra aleatoria representativa de la población. (Martínez, 2006).

a) Participantes setenta alumnos de primer grado de secundaria de edades comprendidas entre los 12 años con 8 meses los menores y 14 años con 9 meses la persona mayor.

b) Instrumentos: auto-reporte, en el cual respondieron cuestionamientos que miden, mediante la escala de Likert (la cual se integró a partir de la información que se obtuvo en la primera fase del proceso), 4 dimensiones de la competencia lectora.

Para validar la consistencia interna del instrumento, se utilizó el procedimiento coeficiente alfa de Cronbach, para lo cual se calculó el promedio de las correlaciones existentes entre los ítems que depositan al concepto oculto que se midió (Barraza, 2007).

c) Procedimientos de colección de datos:

1. Se seleccionó al grupo de participantes mediante una muestra aleatoria.

2. Se invitó a los alumnos a participar de esta prueba de investigación.

3. Se aplicó al grupo el instrumento de auto-reporte, en formato impreso.

4. La recolección de datos se llevó a cabo mediante un análisis psicométrico, para lo cual se utilizó una plantilla de Excel en donde se capturaron los datos colectados.

d) Procedimientos de análisis de datos: el análisis de datos se apoyó del análisis psicométrico, logrando de manera expedita la obtención de la información cuantitativa que el estudio requería.

\section{Resultados}

Etapa 1: análisis de datos cualitativos 
En la tabla 1 se ofrecen datos relacionados con los alumnos entrevistados en la etapa cualitativa. Dado que la pregunta motivo de la investigación plantea analizar las características de los alumnos competentes en lectura que cursan el primer año, se recurrió a entrevistar a tres alumnos de dicho nivel educativo y tres del siguiente nivel inmediato superior.

Tabla 4. Datos generales de los alumnos entrevistados

\begin{tabular}{lccc}
\hline Alumno & Edad & Género & Grado \\
\hline 1 & $13 / 3$ & $\mathrm{H}$ & $1^{\circ}$ \\
\hline 2 & $13 / 6$ & $\mathrm{M}$ & $2^{\circ}$ \\
\hline 3 & $12 / 10$ & $\mathrm{H}$ & $1^{\circ}$ \\
\hline 4 & $13 / 6$ & $\mathrm{M}$ & $2^{\circ}$ \\
\hline 5 & $13 / 1$ & $\mathrm{M}$ & $1^{\circ}$ \\
\hline 6 & $13 / 8$ & $\mathrm{H}$ & $2^{\circ}$
\end{tabular}

Destacan las razones por las cuales los alumnos entrevistados se asumen a sí mismos como lectores competentes (autoconcepto), cuáles son las habilidades que identifican a partir de sí como propias del desarrollo de la competencia lectora que ellos han perfeccionado (habilidades implicadas) y en qué aspectos de la vida cotidiana les ha sido útil ser lectores competentes (utilidad práctica). Ver tabla 2.

Tabla 2. Autoconcepto de competencia lectora, habilidades y utilidad

\begin{tabular}{|c|c|c|c|}
\hline Alumno & Autoconcepto & Habilidades implicadas & Utilidad práctica \\
\hline 1 & $\begin{array}{l}\text { Afirma tener } \\
\text { experiencia lectora al } \\
\text { leer claro y fuerte, tener } \\
\text { pocos errores, gozar de } \\
\text { la lectura y } \\
\text { comprenderla. }\end{array}$ & $\begin{array}{l}\text { Leer rápido, fuerte, claro, } \\
\text { con intensidad; de acuerdo } \\
\text { con el tipo de texto } \\
\text { (contextualizando). } \\
\text { Respetar los signos de } \\
\text { puntuación. }\end{array}$ & $\begin{array}{l}\text { Participar activamente en } \\
\text { actividades religiosas, } \\
\text { escolares y familiares ya que } \\
\text { es un referente reconocido } \\
\text { en sendos espacios sociales. } \\
\text { Obtener resultados positivos } \\
\text { en actividades escolares. }\end{array}$ \\
\hline 2 & $\begin{array}{l}\text { Ha leído varios libros. } \\
\text { Para su edad, posee un } \\
\text { amplio vocabulario y } \\
\text { está segura de tener }\end{array}$ & $\begin{array}{l}\text { Leer frecuentemente. } \\
\text { Conocer sobre materias y } \\
\text { temas } \\
\text { Improvisar historias con }\end{array}$ & $\begin{array}{l}\text { Incremento en la seguridad } \\
\text { para la vida social. Mayor } \\
\text { interacción con los } \\
\text { miembros de la familia. }\end{array}$ \\
\hline
\end{tabular}




\begin{tabular}{|c|c|c|c|}
\hline & $\begin{array}{l}\text { más conocimientos por } \\
\text { ello. }\end{array}$ & facilidad. & $\begin{array}{l}\text { Resultados } \\
\text { exitosos. }\end{array}$ \\
\hline 3 & $\begin{array}{l}\text { Disfruta la lectura y } \\
\text { afirma poseer un alto } \\
\text { grado de concentración. } \\
\text { Reconoce su progreso } \\
\text { al hacer lecturas cada } \\
\text { vez más complicadas. }\end{array}$ & $\begin{array}{l}\text { Comprensión textual, } \\
\text { concentración, desarrollo } \\
\text { de gustos temáticos y de } \\
\text { género variados. }\end{array}$ & $\begin{array}{l}\text { Socializar con personas que } \\
\text { gustan de la lectura. Gozar } \\
\text { de reconocimiento familiar } \\
\text { debido a los conocimientos } \\
\text { adquiridos mediante la } \\
\text { lectura. Crearse criterio } \\
\text { propio. }\end{array}$ \\
\hline 4 & $\begin{array}{l}\text { Comprende las lecturas, } \\
\text { lee con fluidez. Sabe } \\
\text { distinguir cuando tiene } \\
\text { desinterés por una } \\
\text { lectura. }\end{array}$ & $\begin{array}{l}\text { Fluidez y comprensión } \\
\text { lectoras. Legítimo interés } \\
\text { por la lectura. }\end{array}$ & $\begin{array}{l}\text { Académicamente aplicando } \\
\text { los conocimientos obtenidos. } \\
\text { Socialmente al tener } \\
\text { capacidad de generar } \\
\text { opinión propia. }\end{array}$ \\
\hline 5 & $\begin{array}{l}\text { Asevera que lee con } \\
\text { fluidez, posee un } \\
\text { amplio vocabulario de } \\
\text { acuerdo con su edad, } \\
\text { comprende de } \\
\text { inmediato sin necesidad } \\
\text { de detenerse o releer. }\end{array}$ & $\begin{array}{l}\text { Leer con fluidez y } \\
\text { comprensión, alto grado } \\
\text { de concentración, pericia } \\
\text { en la búsqueda de } \\
\text { significados, dedicación. }\end{array}$ & $\begin{array}{l}\text { Le ha favorecido en la vida } \\
\text { social al conocer, interactuar } \\
\text { y conversar con otras } \\
\text { personas. Aprender a través } \\
\text { de la lectura aspectos de la } \\
\text { naturaleza humana. }\end{array}$ \\
\hline 6 & $\begin{array}{l}\text { Asegura que lee } \\
\text { completamente un libro } \\
\text { de manera mensual y es } \\
\text { la persona que más lee } \\
\text { en su familia. }\end{array}$ & $\begin{array}{l}\text { Leer y gozar de la lectura } \\
\text { veloz, y además me gusta } \\
\text { leer rápido. Destreza en la } \\
\text { comprensión del } \\
\text { contenido. }\end{array}$ & $\begin{array}{l}\text { Tener la capacidad de } \\
\text { expresarse amplia y } \\
\text { claramente en diferentes } \\
\text { ámbitos de la vida. Ser una } \\
\text { persona culta. }\end{array}$ \\
\hline
\end{tabular}

De acuerdo con la pregunta de investigación planteada se establece una serie de atributos proporcionados por los niños entrevistados (Tabla 3).

Tabla 3. Atributos que distinguen a alumnos con alto nivel de competencia lectora

\begin{tabular}{ll}
\hline Atributo & Descripción \\
\hline $\begin{array}{l}\text { Edad en la que se } \\
\text { iniciaron en la lectura }\end{array}$ & La generalidad establece que entre los 7 y los 9 años de edad. \\
\hline $\begin{array}{l}\text { Forma en la que se } \\
\text { iniciaron en la lectura }\end{array}$ & $\begin{array}{l}\text { Motivados por un familiar: esencialmente los padres aunque también } \\
\text { los hermanos y primos. }\end{array}$ \\
\hline Decisión de realizar & $\begin{array}{l}\text { Los alumnos han desarrollado la capacidad de decidir qué lecturas } \\
\text { hacer y en qué momentos realizarlas. Se apoyan esencialmente de }\end{array}$ \\
\hline
\end{tabular}


una lectura recomendaciones personales aunque también recurren a las formales: sinopsis.

Mantenerse atraído a La mayoría refiere llegar al final de la lectura una vez que ha decidido una lectura realizarla. Suelen abandonarlas cuando no muestran significantes personales.

Comentar las lecturas Intercambiar opiniones con padres y compañeros.

Interés por desarrollar Realizar lecturas que implican mayores retos de conocimientos y más la competencia habilidades lectoras. / No suspender el hábito lector / Abrirse a la lectora diversidad de la oferta literaria

Leer con frecuencia, Generalmente los alumnos leen diariamente durante al menos una duración. hora, hasta llegar a leer tres horas. Como mínimo leen tres veces a la semana.

Etapa 2: análisis de datos cuantitativos

Análisis descriptivo.

En la tabla 4 se muestra un resumen de los resultados generales obtenidos por cada uno de los 10 reactivos de cada dimensión, incluyendo el promedio que resulta de cada dimensión, así como el promedio general que surge de las 4 dimensiones estudiadas, referentes a la media, los valores mínimos y máximos, los rangos estadísticos, la desviación estándar y el coeficiente de asimetría.

Los resultados promedio muestran que los alumnos poseen una media de 3.04 puntos, el rango menor fue reportado en 1.75 y el mayor en 3.75 puntos en promedio. La mesocompetencia con menor puntuación media fue la segunda, referente a la capacidad para realizar interpretaciones o inferencias directas, la cual se reporta con 3.00 puntos, mientras que la mesocompetencia con mayor puntuación media fue la tercera, cuyo contenido se refiere a la capacidad para hacer valoraciones, interpretar e integrar ideas e informaciones, en la cual se obtuvo una puntuación de 3.08 puntos.

En lo referente a la desviación estándar, la cual determina el grado de homogeneidad del grupo de personas con el cual se trabajó, los resultados promedio muestran 0.46 puntos de desviación estándar, una homogeneidad media. La dimensión con mayor heterogeneidad fue la segunda, referente a la realización de inferencias directas, la cual reportó un resultado de 0.57 puntos. 
Tabla 4. Resumen de datos descriptivos obtenidos

\begin{tabular}{|c|c|c|c|c|c|c|}
\hline Reactivo & Media & $\begin{array}{l}\text { Valor } \\
\text { mínimo }\end{array}$ & $\begin{array}{l}\text { Valor } \\
\text { máximo }\end{array}$ & Rango & $\begin{array}{l}\text { Desviación } \\
\text { estándar }\end{array}$ & $\begin{array}{l}\text { Coef. de } \\
\text { asimetría }\end{array}$ \\
\hline R01 & 3.29 & 1.00 & 4.00 & 3.00 & 0.87 & -1.28 \\
\hline R02 & 2.89 & 1.00 & 4.00 & 3.00 & 0.94 & -0.52 \\
\hline R03 & 2.77 & 1.00 & 4.00 & 3.00 & 1.01 & -0.39 \\
\hline R04 & 3.00 & 1.00 & 4.00 & 3.00 & 0.76 & -0.41 \\
\hline R05 & 2.70 & 1.00 & 4.00 & 3.00 & 0.80 & -0.08 \\
\hline R06 & 3.43 & 1.00 & 4.00 & 3.00 & 0.83 & -1.27 \\
\hline R07 & 3.56 & 2.00 & 4.00 & 2.00 & 0.65 & -1.19 \\
\hline R08 & 2.67 & 1.00 & 4.00 & 3.00 & 0.83 & -0.26 \\
\hline R09 & 3.11 & 1.00 & 4.00 & 3.00 & 0.81 & -0.72 \\
\hline R10 & 3.20 & 1.00 & 4.00 & 3.00 & 0.84 & -0.84 \\
\hline Promedio & 3.06 & 1.80 & 3.80 & 2.00 & 0.43 & -0.71 \\
\hline R11 & 3.29 & 1.00 & 4.00 & 3.00 & 0.85 & -1.02 \\
\hline R12 & 2.57 & 1.00 & 4.00 & 3.00 & 0.97 & -0.21 \\
\hline R13 & 3.01 & 1.00 & 4.00 & 3.00 & 1.08 & -0.73 \\
\hline R14 & 2.81 & 1.00 & 4.00 & 3.00 & 0.98 & -0.37 \\
\hline R15 & 2.97 & 1.00 & 4.00 & 3.00 & 0.78 & -0.52 \\
\hline R16 & 2.54 & 1.00 & 4.00 & 3.00 & 1.05 & -0.08 \\
\hline R17 & 3.10 & 1.00 & 4.00 & 3.00 & 0.95 & -0.62 \\
\hline R18 & 3.26 & 1.00 & 4.00 & 3.00 & 0.94 & -0.97 \\
\hline R19 & 3.00 & 1.00 & 4.00 & 3.00 & 0.88 & -0.52 \\
\hline $\mathbf{R} 20$ & 3.44 & 1.00 & 4.00 & 3.00 & 0.81 & -1.16 \\
\hline Promedio & 3.00 & 1.60 & 3.90 & 2.30 & 0.57 & -0.85 \\
\hline R21 & 3.04 & 1.00 & 4.00 & 3.00 & 0.88 & -0.62 \\
\hline R22 & 3.11 & 1.00 & 4.00 & 3.00 & 0.89 & -0.98 \\
\hline R23 & 3.63 & 2.00 & 4.00 & 2.00 & 0.64 & -1.52 \\
\hline $\mathbf{R} 24$ & 3.49 & 1.00 & 4.00 & 3.00 & 0.72 & -1.28 \\
\hline R25 & 2.91 & 1.00 & 4.00 & 3.00 & 0.88 & -0.62 \\
\hline $\mathbf{R} 26$ & 3.07 & 1.00 & 4.00 & 3.00 & 0.82 & -0.46 \\
\hline $\mathbf{R} 27$ & 2.93 & 1.00 & 4.00 & 3.00 & 0.91 & -0.22 \\
\hline $\mathbf{R} 28$ & 3.07 & 1.00 & 4.00 & 3.00 & 0.89 & -0.65 \\
\hline $\mathbf{R} 29$ & 2.97 & 1.00 & 4.00 & 3.00 & 0.98 & -0.71 \\
\hline R30 & 2.61 & 1.00 & 4.00 & 3.00 & 1.11 & -0.23 \\
\hline Promedio & 3.08 & 1.70 & 3.90 & 2.20 & 0.49 & -0.87 \\
\hline R31 & 3.07 & 1.00 & 4.00 & 3.00 & 0.82 & -0.78 \\
\hline R32 & 2.90 & 1.00 & 4.00 & 3.00 & 1.07 & -0.61 \\
\hline R33 & 3.13 & 1.00 & 4.00 & 3.00 & 0.88 & -0.52 \\
\hline R34 & 2.49 & 1.00 & 4.00 & 3.00 & 0.96 & -0.11 \\
\hline R35 & 3.36 & 1.00 & 4.00 & 3.00 & 0.92 & -1.24 \\
\hline R36 & 3.31 & 1.00 & 4.00 & 3.00 & 0.84 & -1.11 \\
\hline R37 & 2.97 & 1.00 & 4.00 & 3.00 & 0.88 & -0.59 \\
\hline R38 & 3.06 & 1.00 & 4.00 & 3.00 & 0.96 & -0.72 \\
\hline R39 & 3.07 & 1.00 & 4.00 & 3.00 & 0.91 & -0.63 \\
\hline R40 & 2.64 & 1.00 & 4.00 & 3.00 & 1.17 & -0.33 \\
\hline Promedio & 3.00 & 1.60 & 3.80 & 2.20 & 0.56 & -0.63 \\
\hline $\begin{array}{l}\text { Promedio } \\
\text { global }\end{array}$ & 3.04 & 1.75 & 3.75 & 2.00 & 0.46 & -0.81 \\
\hline
\end{tabular}

Por lo que respecta al resultado del coeficiente de asimetría, el cual hace una caracterización del grado de asimetría con respecto a la media, se obtuvieron resultados promedio de la competencia lectora, que muestran un promedio de -0.81 puntos. A partir de los valores máximo y mínimo (1.75-3.75) se establecieron 5 clases de 0.4 puntos de tamaño. 
La población de las clases va en sentido creciente conforme crece el nivel de dominio de la competencia. La primera clase es la más pequeña, con 4 alumnos mientras que la más amplia es la quinta con 22 alumnos. La mayoría de los encuestados (22 alumnos) entonces, se ubica en la quinta clase, en la que se encuentra una frecuencia de 3.35 a 3.75 puntos. El mayor nivel de dominio de la competencia, por lo tanto, lo ostentan 22 alumnos. Estos datos se muestran en los resultados vertidos por el histograma de la gráfica 1.

Gráfica 1. Histograma global de la competencia lectora

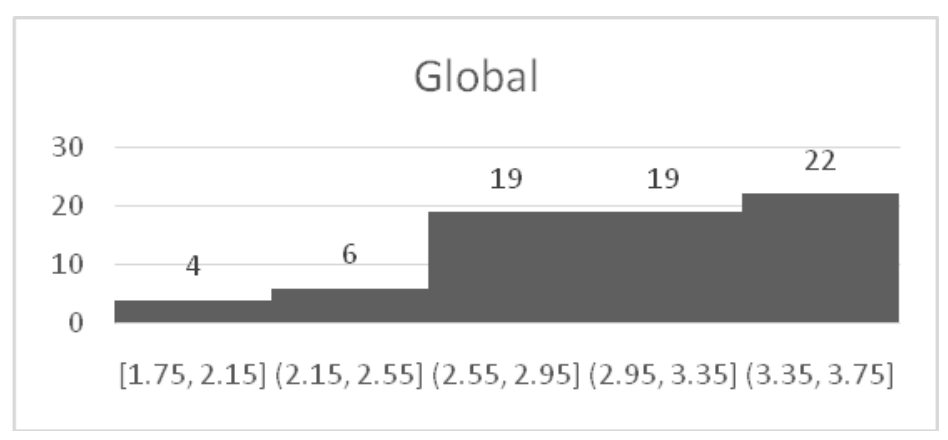

Análisis psicométrico

Por lo que respecta al nivel de confiabilidad, se muestra el resultado obtenido después de calcular los valores del coeficiente alfa de Cronbach para cada dimensión y para el instrumento en general, como se muestra en la tabla 5:

Tabla 5. Resultados de confiabilidad del instrumento

\begin{tabular}{lllll}
\hline Escala 1 & Escala 2 & Escala 3 & Escala 4 & Global \\
\hline 0.677 & 0.813 & 0.751 & 0.794 & 0.927 \\
\hline
\end{tabular}

En lo referente a la validez de constructo, fue preciso realizar el análisis de pertenencia de cada reactivo para la escala a la que fue diseñado. Así, se determinaron las correlaciones de cada reactivo con el promedio de cada dimensión, así como el promedio de cada dimensión con el de las otras tres dimensiones.

Por lo que respecta a las escalas 2 y 4 , estas muestran correlaciones altas con respecto al resto de las escalas, es decir, sus puntuaciones son mayores para los reactivos correspondientes. Esto significa que los reactivos están bien construidos y que son pertinentes a la escala para la que fueron construidos.

La escala 1 muestra el mayor número de imprecisiones en cuanto a la elaboración de los reactivos o de su ubicación en la escala, la escala 3 también muestra un error en el reactivo 27 , el cual no correlaciona con la escala a la que fue diseñado, sino a la escala 1.

Análisis de relaciones 
a) Entre los promedios de las distintas dimensiones, es decir, las correlaciones existentes de la escala 1 con las escalas 2, 3 y 4, de la 2 con la 1, 3 y 4 y así sucesivamente. Esto se establece mediante un estudio de coeficiente de correlación, tal como se muestra en la tabla 6 .

Tabla 6. Correlaciones entre escalas

\begin{tabular}{|l|r|r|r|r|}
\hline & Escala 1 & Escala 2 & Escala 3 & Escala 4 \\
\hline Escala 1 & 1.00 & 0.78 & 0.78 & 0.64 \\
\hline Escala 2 & 0.78 & 1.00 & 0.76 & 0.70 \\
\hline Escala 3 & 0.78 & 0.76 & 1.00 & 0.81 \\
\hline Escala 4 & 0.64 & 0.70 & 0.81 & 1.00 \\
\hline
\end{tabular}

En la tabla 6 se puede observar que la relación de cada escala consigo misma es de 1, las demás correlaciones, aunque se establecen a la baja con respecto a sí mismas, existe un empalme entre ellas, lo que significa que las escalas no miden relaciones independientes, sino que son muy cercanas entre sí y los reactivos no precisan su pertenencia a la escala para la que fueron diseñados.

a) Variable demográfica. Comparación de medias entre género.

Tabla 7. Variables demográficas de género

\begin{tabular}{|c|c|c|c|c|c|c|}
\hline \multirow{2}{*}{$\begin{array}{l}\text { Género } \\
\text { Hombres }\end{array}$} & \multirow[t]{2}{*}{ - Escala 1} & \multirow{2}{*}{$3.02^{\text {Escala 2 }}$} & \multirow{2}{*}{$2.94^{\text {Escala 3 }}$} & \multirow{2}{*}{$3.05^{\text {Escala 4 }}$} & \multicolumn{2}{|c|}{ Promedio de global } \\
\hline & & & & & 3.00 & 3.00 \\
\hline Mujeres & & 3.17 & 3.14 & 3.16 & 3.00 & 3.12 \\
\hline Total ge & & 3.06 & 3.00 & 3.08 & 3.00 & 3.04 \\
\hline
\end{tabular}

Se observa que en las tres primeras escalas las mujeres muestran mayor grado de dominio de las mesocompetencias lectoras y el mismo grado de dominio en la escala 4. Por tal motivo, en los resultados globales las mujeres se encuentran por encima de la media reportada.

\section{Discusión}

Los expertos en materia de competencia lectora han descrito cuáles son las necesidades, características y posibilidades de desarrollo de las personas competentes en lectura. En el estudio fue posible atestiguar, mediante el análisis de diferentes investigaciones en materia lectora, cuáles son las condiciones, las problemáticas y las políticas y programas de atención para el desarrollo de la competencia lectora. Se ha presentado una identificación y caracterización del comportamiento de los buenos lectores, el cual puede resultar útil al momento de identificar el nivel de dominio de la competencia lectora en alumnos de secundaria.

La investigación permitió conocer las particularidades que hacen de una persona ser competente en lectura. En un segundo momento, dio pauta para saber el nivel de dominio de la competencia que posee la comunidad escolar a la que pertenecen las personas entrevistadas.

El estudio puede contribuir a medir dos momentos concretos: si la escuela pretende implementar un programa que desarrolle la competencia lectora, el método utilizado podría 
ser útil para medir el nivel de dominio antes de empezar el programa y en un segundo momento, después de haber llevado a cabo el programa. La estrategia puede ser exitosa, a pesar de las deficiencias que el instrumento utilizado reportó al momento de analizar las correlaciones entre las escalas propuestas para medir el dominio de las mesocompetencias propuestas y que es perfectible. Los resultados obtenidos permiten afirmar que el estudio puede aplicarse a otros niveles educativos, como en primaria, bachillerato e incluso a nivel universitario. Puede dar buenos resultados de investigación en otros lugares, tanto del país como de países de habla hispana. El método es aplicable a otros contextos, en este caso a otras competencias si se trabaja adecuadamente un instrumento en particular.

Una de las principales dificultades que surgieron durante la investigación fue que no se tenía la certeza de lograr la identificación correcta de los alumnos que participaron en la etapa cualitativa. Implicó un trabajo minucioso dado que se recurrió a verificar opiniones diversas y a establecer diálogos informales con una serie de candidatos hasta asegurar que se contaba con los estudiantes idóneos.

Por lo que respecta a la puesta en práctica de la propuesta para medir el nivel de dominio de la competencia lectora, se sugiere delimitar los rangos de acción de cada escala de dimensión para que tengan mayor precisión en cuanto a su diferenciación y por ende al nivel de competencia que miden.

También se sugiere aplicar la técnica test retest, para verificar la confiabilidad del instrumento. Además, se propone operar la medición a la par de la puesta en operación de un programa de fomento al desarrollo de la competencia lectora.

\section{Referencias bibliográficas}

Barraza, A. (2007). Apuntes sobre metodología de la investigación. ¿Confiabilidad? Investigación Educativa Duranguense. (6), 6-10. Recuperado el 7 de mayo de 2013 de: http://www.upd.edu.mx/librospub/revistas/invedu06.pdf

Fundación Este País (2011). Resultados de la prueba PISA de la OCDE. Este país. Tendencias y opiniones, (237) 61-64. Recuperado el 23 de abril de 2013 de: http://estepais.com/site/wp-content/uploads/2011/01/17_fep_resultadospisa_237.pdf

FunLectura (2012). De la penumbra a la oscuridad... Encuesta Nacional de Lectura 2012. Primer Informe. Recuperado el 23 de abril de 2013 de: http://www.caniem.org/Archivos/funlectura/EncuestaNacionaldeLectura2012/Encuest aNacionaldeLectura2012.html

Instituto Nacional de Evaluación Educativa. (2012). PIRLS: Estudio Internacional de Progreso en Comprensión Lectora. Madrid, España: Ministerio de Educación, Cultura y Deporte. Recuperado el 3 de febrero de 2013 de: http://www.mecd.gob.es/inee/estudios/pirls.html 
León, J.A., Escudero, I. y Olmos, R. (2012). Evaluación de la comprensión lectora. Manual. Madrid, España: Tea ediciones. Recuperado el 2 de febrero de 2013 de: http://www.web.teaediciones.com/Ejemplos/ECOMPLEC_MANUAL_WEB.pdf

Martínez, P. C. (2006). El método de estudio de caso. Estrategia metodológica de la investigación científica. Pensamiento y gestión. (20), 165-193. Recuperado el 6 de febrero de 2013 de:

http://ciruelo.uninorte.edu.co/pdf/pensamiento_gestion/20/5_El_metodo_de_estudio_de_caso. pdf

OCDE (2006). PISA 2006. Marco de la evaluación. Conocimientos y habilidades en ciencias, matemáticas y lectura. Recuperado el 31 de enero de 2013 de: http://www.oecd.org/pisa/39732471.pdf

Pereira, Z. (2011). Los diseños de método mixto en la investigación en educación: una experiencia concreta. Revista Electrónica Educare. XV (1), 15-29. Recuperado el 5 de febrero de 2013 de: http://www.revistas.una.ac.cr/index.php/EDUCARE/article/view/867

Valenzuela, J. R. y Flores, M. (2011). Fundamentos de investigación educativa (eBook). Monterrey, México: Editorial Digital Tecnológico de Monterrey. 